\title{
Joint Ground and Air Emergency Medical Services Coverage Models: A Greedy Heuristic Solution Approach
}

\author{
Elif Tokar Erdemir ${ }^{1}$, Rajan Batta ${ }^{2,3,5}$, \\ Peter A. Rogerson ${ }^{2,4,5}$, Alan Blatt ${ }^{2}$, and Marie Flanigan ${ }^{2}$ \\ 1: Industrial and Systems Engineering Program, University of Minnesota, Minneapolis, MN 55455 \\ 2: Center for Transportation Injury Research, CUBRC, Buffalo, NY 14225 \\ 3: Dept. of Industrial and Systems Engineering, University at Buffalo (SUNY), Buffalo, NY 14260 \\ 4: Dept. of Geography, University at Buffalo (SUNY) \\ 5: National Center for Geographic Information and Analysis, University at Buffalo (SUNY) \\ Corresponding author: Batta; email: batta@buffalo.edu
}

\section{Revised: December 2009}

\begin{abstract}
Aeromedical and ground ambulance services often team up in responding to trauma crashes, especially when the emergency helicopter is unable to land at the crash scene. We propose location coverage models and a greedy heuristic for their solution to simultaneously locate ground and air ambulances, and landing zones (transfer points). We provide a coverage definition based on both response time and total service time, and consider three coverage options; only ground Emergency Medical Services (EMS) coverage, only air EMS coverage, or joint coverage of ground and air EMS in which the patient is transferred from an ambulance into an emergency helicopter at a transfer point. To analyze this complex coverage situation we develop two sets of models which are variations of the Location Set Covering Problem (LSCP) and the Maximal Covering Location Problem (MCLP). These models address uncertainty in spatial distribution of motor vehicle crash locations by providing coverage to a given set of both crash nodes and paths. The models also consider unavailability of ground ambulances by drawing upon concepts from backup coverage models. We illustrate our results on a case study that uses crash data from the state of New Mexico. The case study shows that crash node and path coverage percentage values decrease when ground ambulances are utilized only within their own jurisdiction.
\end{abstract}

Keywords: Emergency services, Trauma crashes, Aeromedical, Location coverage models, Backup coverage

\section{Introduction and Motivation}

Both ground and air ambulances are used in transportation of trauma patients. There are many factors that affect which type of transportation is more advantageous to the seriously injured trauma victim in terms of providing less out-of-hospital time (i.e. the time from the accident's occurrence until reaching the hospital). For example, if the incident scene is close to a trauma center (TC), then ground ambulances are preferred; if the scene is in a rural area far away from a TC, then air ambulances are preferred. In addition, ground and air EMS may work 
together - especially when the incident scene does not have a suitable area nearby for a helicopter to safely land. In this case, a ground ambulance provides initial response at the scene and then transports the patient to the closest transfer point (landing zone) where the patient is transferred to an air ambulance.

In this work, we focus on trauma cases resulting from motor vehicle crashes. As we mentioned in our previous work on locating aeromedical bases (Tokar Erdemir et al., 2008), motor vehicle crash locations are effectively modeled using a combination of crash nodes and crash paths. Crash nodes are the infinitesimal locations in a spatial region where crashes occur frequently, such as main highway intersections. Uncertainty associated with exact crash location is captured through the coverage of crash paths, which are the road segments on which crashes occur.

In practice, the most preferred (nearest) ground ambulance may be busy responding to another emergency when its service is requested. In such cases, the calls are handled by other available ground ambulances. Backup coverage is a method to achieve this. We provide backup coverage by either another ground ambulance or by ensuring coverage from an air ambulance.

The contribution of this paper is summarized as follows:

- Two location-coverage models are proposed for simultaneous location of ground ambulances, air ambulances and transfer points.

- Only ground, only air, and joint ground-air through a transfer point coverage options are considered in the models for both crash nodes and crash paths; where a joint coverage through a transfer point is a new concept in location-coverage literature.

- Coverage is considered as a combination of response time to the scene and total service time until the crash victim is transported to the closest trauma center.

- Unavailability of ground ambulances is addressed through backup coverage.

- For the first model, problem size reduction techniques are introduced, and a greedy heuristic algorithm is proposed for the solution of both models.

- Models are illustrated on a case study that uses motor vehicle crash data and emergency hospital data from the State of New Mexico (NM). Results are provided for different combinations of first and backup coverage weights, and for the case where ground ambulances can only be dispatched to crash locations within their own jurisdiction. 


\section{Literature Review}

We now review some of the literature on EMS location coverage models. The literature on coverage models for emergency services is divided into two major parts: the Location Set Covering Problem (LSCP) and the Maximal Covering Location Problem (MCLP). LSCP was introduced by Toregas et al. (1971) and determines the minimum number of servers (and server locations) required to cover all demand points. However, the number of resources to cover all demand points could be excessive in many cases, and this is addressed by MCLP (Church and ReVelle, 1974). The objective of MCLP is to maximize the total amount of demand served within a maximal coverage distance given a limited budget, (i.e. the number of facilities that can be located is fixed). One of the earlier applications using MCLP is the study by Eaton et al. (1985) to plan the emergency medical service in Austin, Texas. Even with increased calls for service, the paper succeeded in decreasing the average response time by using MCLP.

Neither LSCP nor MCLP considers the situation that requires dispatching vehicles of several types to an incident scene. The Tandem equipment allocation model (TEAM) by Schilling et al. (1979) is one of the earliest models that deals with multiple types of servers. This model is developed for fire companies that operate with two types of equipment: pumpers and rescue ladders. Schilling et al. (1979) also introduced another model, known as FLEET (Facility Location and Equipment Emplacement Technique), in which two different types of servers need to be located. They generalized the MCLP model to locate emergency fire fighting servers and depots in the City of Baltimore. Later, Marianov and ReVelle (1992) developed another model to locate fire stations with limited capacity of pumper and rescue ladders, ensuring each demand point is covered by the required equipment. Branas et al. (2000) introduces a mathematical model named the Trauma Resource Allocation Model for Ambulances and Hospitals (TRAMAH). The model simultaneously locates ambulances and hospitals with an objective of maximizing coverage of severely injured patients. For the solution of TRAMAH, Branas and ReVelle (2001) develop an algorithm that combines a mixed-integer program and an iterative switching heuristic. Lerner et al. (1999) uses GIS and historical transport data to identify zones from which only ground or only air transportation of patients would result in shorter out-of-hospital times without considering joint coverage of ground and air ambulances. We refer the reader to Marianov and ReVelle (1995) and Brotcorne et al. (2003) for a more detailed review of the literature on emergency services location problems.

The original location-coverage models were later extended to ensure backup coverage. Daskin and Stern (1981) modified LSCP to allow for multiple coverage. The disadvantage of this model is that while some demand nodes have many redundant coverers, others could be left with 
only a first coverer. Hogan and ReVelle (1986) corrected this drawback in multiple coverage LSCP by maximizing backup coverage. They introduced two different models, named BACOP1 and BACOP2. BACOP1 maximizes backup coverage while requiring each demand point to have first coverage. BACOP2 relaxes the first coverage requirement and it trades off first coverage against backup coverage by assigning weights to each. The paper by Bianchi and Church (1988) proposes a model named Multiple coverage, One-unit Facility Location and Equipment Emplacement Technique (MOFLEET). This model minimizes the chances that an ambulance will be unavailable when requested by simultaneously ensuring coverage of a demand point by alternative servers.

Another part of the EMS literature focuses on analyzing EMS system performance by utilizing the hypercube queuing model of Larson (1974), which is based on spatially distributed queuing theory and used to analyze server-to-customer systems. Mendonça and Morabito (2001) analyze the ambulance deployment of an emergency medical system on a Brazilian highway. They focus on the mean response time of the system to an emergency call. By applying the hypercube model, they show that the workload imbalance among the ambulances could be reduced. Later, Atkinson et al. $(2006,2008)$ studies the hypercube queuing model with customer-dependent service rates. In this case, the hypercube model has $3^{n}$ states, where $n$ is the number of server bases, meaning that exact solutions for the long-run proportion of lost demands can be obtained only for small-sized problems. The papers introduce heuristic methods to obtain accurate estimates of stationary loss probability. Chelst and Barlach (1981) extend the hypercube model by considering that multiple identical servers are dispatched to each call. They present one exact and one approximate model to capture the simultaneous response of two servers. Recently, Iannoni and Morabito (2007) address the multiple-dispatch problem by considering that emergency calls are of different types, the servers are distinct and only certain servers can service calls in a given region (partial backup).

Demand locations are typically of two varieties - those which have great spatial certainty (e.g. accidents that occur at a historically dangerous intersection) and those which have spatial uncertainty (e.g. accidents that occur in a dangerous 5-mile stretch of road). To capture these two demand location types, Tokar-Erdemir et al. (2008) developed the notion of coverage of demand nodes and demand paths. One of these models was later used by Tokar-Erdemir et al. (2008) to locate aeromedical bases by providing coverage to both demand nodes and paths (which they refer to as crash nodes and crash paths).

The models that we develop in this paper build on features from LSCP, MCLP, backup coverage, and demand location types. Their development is detailed in the next section. 


\section{Preliminaries for Model Development}

The models that we developed address the problem of locating ground and air ambulances, and transfer points (landing zones). We consider three options of sending ground and air EMS to trauma cases:

GROUND: Send only a ground ambulance

AIR: Send only an air ambulance

JOINT GROUND-AIR: Send an ambulance and a helicopter (simultaneous dispatch)

These three options lead to three different coverage definitions respectively. A trauma incident location is covered if and only if:

GROUND COVERED: At least one ground ambulance is located within a pre-specified travel time to the incident location, and it can also take the trauma victim to the closest TC within a pre-specified time $T$ by ground, or

AIR COVERED: At least one air ambulance is located within a pre-specified travel time to the incident location, and it can take the patient to the closest TC within a pre-specified time $T$ by air, if the air ambulance is able to land on to the incident location, or JOINT GROUND-AIR COVERED: At least one ground ambulance-transfer point-air ambulance combination is located within a pre-specified travel time to the incident location and in such a way that the servers can take the patient to the closest TC within a pre-specified time $T$. This coverage option applies when the air ambulance cannot land at the crash scene. The ground ambulance takes the patient to a transfer point where it is met by an air ambulance. The patient is transferred and the air ambulance takes the patient to the TC.

Considering the above three coverage cases, crash locations are classified into two groups: locations where air ambulances can land and locations where air ambulances cannot land. For the former group, we consider only ground or only air coverage. For the latter group, we consider only ground or joint ground-air coverage.

Two models are developed for this problem. In both models, we address spatial uncertainty in crash locations, by providing coverage to both crash nodes and paths. Paths correspond to roads on which trauma crashes occur, and crash nodes can be defined as frequent crash occurrence points on the paths.

We also address the potential unavailability of ground ambulances by utilizing a deterministic backup coverage model, BACOP2 (Hogan and ReVelle, 1986). BACOP2 trades off 
first coverage against backup coverage by assigning weights to each in the objective function. Although at times they can be unavailable because of weather, air ambulances are typically called to the most serious trauma scenes (e.g., motor vehicle crashes), especially if these scenes are far from a trauma center. Ground ambulances respond to many types of medical and less serious trauma incidents as well as responding to a major percentage of trauma incidents. If there is only one ground ambulance in an area, there is often a reluctance to commit the only available vehicle to a lengthy transport out of its service area. Thus backup coverage is needed in high crash density regions, which are covered by only a single ground ambulance or a single combination of ground and air ambulances.

We have now set the stage for presenting the two basic models for joint ground and air ambulance coverage. The first model (in Section 4) is based on LSCP. The second model (in Section 5) is based on MCLP.

\section{Set Cover with Backup Model}

We call our first model the Set Cover with Backup Model (SCBM). The thrust of this model is to determine the cost associated with covering all crash locations using a combination of ground and air ambulances. This provides an upper bound or maximal budget needed to create an optimal EMS system. The next model (Section 5) assumes there is a limited budget.

The major difference between SCBM and the variants of LSCP in the location-coverage literature is that SCBM permits coverage through joint use of each of two facility types through a transfer point as well as through exclusive use of each of these two facility types. On the other hand, variants of LSCP consider either dispatching a single server or dispatching multiple servers directly to meet at the demand location.

The decision variables in SCBM are the locations of ground and air ambulances, and transfer points. The objective is to minimize total cost of locating ground ambulances, air ambulances and transfer points. The constraints are (i) all crash nodes and crash paths are covered at least once, and (ii) backup coverage for the crash nodes and crash paths that are covered by exactly one ground ambulance or by exactly one combination of ground and air ambulances.

As opposed to crash nodes, a crash path may have segments in coverage regions of different EMS servers. For small crash path lengths, there is a greater likelihood of a single EMS server covering the entire crash path. Motivated by this observation, we divide each crash path into small linear segments. This allows us to model both crash nodes and crash paths in a similar manner. 


\subsection{Model Formulation}

Sets:

$M_{A}:$ set of potential ground ambulance locations (index: $a$ )

$M_{H}$ : set of potential air ambulance (helicopter) locations (index: $h$ )

$M_{R}:$ set of potential transfer point locations (index: $r$ )

$N$ : set of all crash nodes (index: $j$ )

$P$ : set of all crash paths (index: $k$ )

Parameters:

$c_{A}:$ cost of locating a ground ambulance

$c_{H}:$ cost of locating an air ambulance

$c_{R}:$ cost of locating a transfer point

$A_{a j}\left(A_{a k}\right)= \begin{cases}1, & \text { if potential ground ambulance location } a \text { covers node } j \text { (path } k \text { ) } \\ 0, & \text { otherwise }\end{cases}$

$A_{h j}\left(A_{h k}\right)=\left\{\begin{aligned} 1, & \text { if potential air ambulance location } h \text { covers node } j \text { (path } k \text { ) } \\ & \text { and if air ambulance can land at node } j(\text { path } k) \\ 0, & \text { otherwise }\end{aligned}\right.$

$A_{a h r j}\left(A_{a h r k}\right)=\left\{\begin{array}{l}1, \quad \text { if potential ground }(a) \text { and air }(h) \text { ambulances, and transfer point location } r \\ \quad \text { covers node } j(\text { path } k) \\ 0, \text { otherwise }\end{array}\right.$

Variables:

$x_{a}= \begin{cases}1, & \text { if a ground ambulance is located at } a \\ 0, & \text { otherwise }\end{cases}$

$y_{h}= \begin{cases}1, & \text { if an air ambulance is located at } h \\ 0, & \text { otherwise }\end{cases}$

$z_{r}= \begin{cases}1, & \text { if a transfer point is located at } r \\ 0, & \text { otherwise }\end{cases}$ 


$$
\begin{aligned}
& u_{j}=\left\{\begin{array}{l}
1, \text { if node/path } j \text { is covered by at least one of the located air ambulances } \\
0, \text { if node/path } j \text { is covered by at least two ground ambulances and/or combinations }
\end{array}\right. \\
& v_{j a}=\left\{\begin{array}{l}
1, \text { if node/path } j \text { is covered by ground ambulance } a \\
0, \text { otherwise }
\end{array}\right. \\
& l_{a h r}=x_{a} y_{h} z_{r}=\left\{\begin{array}{c}
1, \text { if a ground ambulance, air ambulance and a transfer point are located at } a, \\
h \text { and } r \text { respectively } \\
0, \text { otherwise }
\end{array}\right.
\end{aligned}
$$

Formulation: 
Minimize $\left(\sum_{a \in M_{A}} c_{A} x_{a}+\sum_{h \in M_{H}} c_{H} y_{h}+\sum_{r \in M_{R}} c_{R} z_{r}\right)-\sum_{j \in N \cup P} u_{j} \cdot \varepsilon$

s.t.

$$
\begin{array}{ll}
\sum_{h \in M_{H}} A_{h j} y_{h} \geq u_{j} & \forall j \in N \cup P \\
A_{a j} x_{a}+\sum_{h \in M_{H}} \sum_{r \in M_{R}} A_{a h r j} l_{a h r} \geq v_{j a} & \forall j \in N \cup P, \forall a \in M_{A} \\
\sum_{a \in M_{A}} v_{j a}=2 \cdot\left(1-u_{j}\right) & \forall j \in N \cup P \\
x_{a} \geq l_{a h r} & \forall a \in M_{A}, h \in M_{H}, r \in M_{R} \\
y_{h} \geq l_{a h r} & \forall a \in M_{A}, h \in M_{H}, r \in M_{R} \\
z_{r} \geq l_{a h r} & \forall a \in M_{A}, h \in M_{H}, r \in M_{R} \\
x_{a}+y_{h}+z_{r}-l_{a h r} \leq 2 & \forall a \in M_{A}, h \in M_{H}, r \in M_{R} \\
x_{a} \in\{0,1\} & \forall a \in M_{A} \\
y_{h} \in\{0,1\} & \forall h \in M_{H} \\
z_{r} \in\{0,1\} & \forall r \in M_{R} \\
l_{a h r} \in\{0,1\} & \forall a \in M_{A}, h \in M_{H}, r \in M_{R} \\
u_{j} \in\{0,1\} & \forall j \in N \cup P \\
v_{j a} \in\{0,1\} & \forall j \in N \cup P, a \in M_{A}
\end{array}
$$

(1) is the objective function. The summation in parenthesis is the total cost of locating ground ambulances, air ambulances, and transfer points. We subtract the sum of variables $u_{j}$ (multiplied by a very small number $\varepsilon$ ) from the total cost. This relaxes the assignment of two different ground ambulances to cover node/path $j$, if there is at least one air ambulance covering $j$. Constraint sets (2), (3) and (4) are the set cover with backup coverage constraints. These constraints ensure that all crash nodes and paths are covered at least once by an air ambulance, or twice by a ground ambulance and/or a combination of ground and air ambulances. Constraint sets (5), (6), (7) and (8) are linearization constraints. Constraint sets (5), (6) and (7) ensure that $l_{a h r}$ cannot be 1 , when at least one of $x_{a}, y_{h}$ or $z_{r}$ is 0 . This means that when at least one of the EMS servers that should be in the combination is not located, then there is no such combination of ground and air ambulances used for service. Constraint set (8) ensures that if all $x_{a}, y_{h}$ and 
$z_{r}$ are 1 , then $l_{a h r}$ cannot be 0 . This means if all the EMS servers that form the combination are located, then this combination should be available to cover the crash nodes and paths in its coverage area.

\subsection{Solution Method}

Since the LSCP is a special case of SCBM and the LSCP is known to be NP-complete (Karp, 1972), we focus on the development of a heuristic method for SCBM as opposed to exact solution methods. In particular, we develop a greedy heuristic approach for solving larger instances of SCBM and also explore the use of ILOG's CPLEX to solve smaller instances of SCBM to optimality. The heuristic's performance is tested by comparing the greedy heuristic results to optimal solutions obtained by CPLEX and premature CPLEX (modified parameter settings).

Considering both single server and joint coverage through a transfer point, and also incorporating backup coverage and path coverage significantly increases the complexity of the problem. Thus, even greedy heuristic runtimes are very long for actual-sized problems. Therefore, this paper focuses on introducing a greedy heuristic for both models and leave development of more sophisticated heuristics as a future work.

Since crash nodes and crash paths can be covered by three different ways (ground, air, joint ground-air), we use the index $i$ to reflect all of these three ways of coverage. Each value of index $i$ corresponds to a unique instance of coverage. For example, $i=10$ could imply coverage provided by a ground ambulance at location 3 (only ground), $i=11$ could imply coverage provided by an air ambulance at location 1 (only air), and $i=12$ could imply coverage provided by a combination of ground ambulance at location 3, air ambulance at location 1 and transfer point at location 8 (joint ground-air).

\section{Greedy Heuristic Parameters:}

- $G$ : Greedy output, i.e. final locations of the air and ground ambulances and transfer points

- $w(i)$ : Function that calculates the number of crash nodes and paths that are covered by combination $i$

- $P_{i}$ : Set of crash nodes and paths that are covered by combination $i$

- $c_{i}$ : Total facility cost of combination $i$. If $i$ refers to a ground (air) ambulance, then $c_{i}$ is the cost of ground (air) ambulance. If $i$ refers to a combination of ground ambulance, air ambulance and transfer point, then $c_{i}$ is the sum of their costs. 
- $C C_{j}=\left\{\begin{array}{cl}0, & \text { if } j \text { is fully covered } \\ 0.5, & \text { if } j \text { is covered just once and with a ground ambulance or combination } \\ 1, & \text { if } j \text { is uncovered }\end{array}\right.$

Here, "fully covered" means that node/path $j$ is either covered by at least one air ambulance, or at least two different ground ambulances or combinations.

We note that the benefit of including combination $i$ in the optimal set of EMS server locations increases with the ratio $\frac{\left|P_{i}\right|}{c_{i}}$ (the number of crash nodes and paths that are covered by $i$ per unit cost). This fact is the driving force behind the greedy heuristic.

Greedy Heuristic Algorithm:

Step 0. Set $G=\varnothing, w(G)=0$, and $C C_{j}=1$ for all $j \in N \cup P$, where $\varnothing$ is the empty set.

Step 1. If $P_{i}=\varnothing$ for all $i \in M_{A} \cup M_{H} \cup M_{A \cdot H \cdot R}$, then stop; $G$ is a cover.

Otherwise,

For all $i \in M_{A} \cup M_{H} \cup M_{A \cdot H \cdot R}$, compute

$$
\operatorname{ratio}(i)=\frac{w(i)}{c_{i}}
$$

where $w(i)=f$ (Nodes and paths weighted by $C C_{j}$ and that are covered by locating combination $i$ )

Evaluation of function $f$ changes with $i$ being a ground ambulance, air ambulance, or a combination of the two. If $i$ is a ground ambulance or a combination, then only half of the crash node or crash path is considered as covered due to backup need.

Let $i^{*}$ be the combination that maximizes $\operatorname{ratio}(i)$ :

$$
i^{*}=\arg \max \operatorname{ratio}(i)
$$

Step 2. Add $i^{*}$ to $G$.

Update location decision variables for $i^{*}$.

If $i^{*}$ is an air ambulance

Delete the crash nodes and paths that are covered by air ambulance $i^{*}$

Set $C C_{j}=0$ for every $j$ covered by air ambulance $i^{*}$

If $i^{*}$ is a ground ambulance,

For uncovered crash nodes/paths $j$ that are covered by locating $i^{*}$, 


\section{Set $C C_{j}$ to 0.5 .}

Record the ground ambulance index that provides first coverage to $j$.

For crash nodes/paths $j$ with only first coverage and that are re-covered by locating ground ambulance $i^{*}$

Set $C C_{j}$ to 0 if first covering ground ambulance is different than $i^{*}$

Delete the covered nodes and paths.

If $i^{*}$ is a combination of an air ambulance and a ground ambulance,

Then the method is the same as shown above for $i^{*}$ being a ground ambulance.

\section{Return to Step 1.}

Before applying the greedy heuristic procedure to a problem instance, we first reduce the size of the problem instance by applying suitable modifications of the row and column elimination methods discussed in Daskin (1995) and in Church and Gerrard (2003) for the LSCP and multi-level LSCP, respectively. This reduction in problem size speeds up the Greedy heuristic and also improves the efficiency of the CPLEX implementation that we later test. The techniques that we used are as follows:

- $\quad$ If $\sum_{i} A_{i j}=1$ (i.e. the number of coverages given to node/path $j$ over all potential combination locations) and if $i^{*}$ (the only combination that covers $j$ ) is a single air ambulance, then $y_{i^{*}}=1$. We eliminate the backup coverage constraints for $j$ and all other rows including $y_{i^{*}}$ from the problem (since no backup coverage is needed for the crash nodes and paths that are covered exactly once and by an air ambulance).

- If $\sum_{i} A_{i j}=2$ and $i_{1}^{*}$ and $i_{2}^{*}$ (the only two servers that cover $j$ ) are both not an air ambulance, then $x_{i_{1}^{*}}$ (or $\left.l_{i_{1}^{*}}\right)=1$ and $x_{i_{2}^{*}}\left(\right.$ or $\left.l_{i_{2}^{*}}\right)=1$. We eliminate all the rows of backup coverage constraints that include $i_{1}^{*}$ and $i_{2}^{*}$ together. Here, $i_{1}^{*}$ and $i_{2}^{*}$ should include different ground ambulances, otherwise the problem becomes infeasible.

We note that several other column reductions were attempted but were not successful due to additional complexity of the problem. 


\section{Maximal Cover for a Given Budget Model}

We now introduce the second model called the Maximal Cover for a Given Budget Model (MCGBM). In this model, we want to find the optimum mix of ground and air ambulances, and transfer points that maximize a weighted combination of first coverage for all crash nodes and paths, and backup coverage for the crash nodes and paths that are covered exactly once by ground or joint ground-air. The numbers of each EMS server to be located are not given separately. Instead, there is a limited budget and we look for maximizing coverage by allocating this budget among the ground ambulances, air ambulances and transfer points optimally. We note that the optimum facility location cost of SCBM is an upper limit on the budget specified in MCGBM. In other words, with MCGBM budget values higher than the optimum SCBM budget, we cannot improve on the total number of covered nodes and paths.

\subsection{Model Formulation}

The new parameters of MCGBM in addition to the ones in SCBM are, $B$ : Maximum budget to be allocated among the EMS servers

$d_{j}$ : Weight attached to node/path $j$

$\theta$ : Weight of first coverage (between 0 and 1) (1- $\theta$ is the weight given to backup coverage)

The new variables are,

$$
\begin{aligned}
& f_{j}=\left\{\begin{array}{l}
1, \text { if node/path } j \text { is covered at least once } \\
0, \text { otherwise }
\end{array}\right. \\
& b_{j}=\left\{\begin{array}{l}
1, \text { if backup coverage is given to node/path } j \\
0, \text { otherwise }
\end{array}\right.
\end{aligned}
$$

$g_{j}=u_{j} b_{j}=\left\{\begin{array}{l}1, \text { if backup coverage is not needed for node/path } j \text { by locating at least one air } \\ \text { ambulance that covers } j \\ 0, \text { otherwise }\end{array}\right.$ 


$$
\begin{aligned}
& \text { Maximize }\left(\theta \sum_{j \in N \cup P} d_{j} f_{j}+(1-\theta) \sum_{j \in N \cup P} d_{j} b_{j}\right)+\varepsilon \cdot \sum_{j \in N \cup P} u_{j} \\
& \text { s.t. } \\
& \sum_{a \in M_{A}} c_{A} x_{a}+\sum_{h \in M_{H}} c_{H} y_{h}+\sum_{r \in\left\{M_{R}-N_{1}\right\}} c_{R} z_{r} \leq B \\
& \sum_{a \in M_{A}} A_{a j} x_{a}+\sum_{h \in M_{H}} A_{h j} y_{h}+\sum_{a \in M_{A}} \sum_{h \in M_{H}} \sum_{r \in M_{R}} A_{a h r j} l_{a h r} \geq f_{j} \quad \forall j \in N \cup P \\
& \sum_{h \in M_{H}} A_{h j} y_{h} \geq g_{j} \\
& A_{a j} x_{a}+\sum_{h \in M_{H}} \sum_{r \in M_{R}} A_{a h r j} l_{a h r} \geq v_{j a} \\
& \forall j \in N \cup P \\
& \forall j \in N \cup P \\
& \sum_{a \in M_{4}} v_{j a}=2 b_{j}-2 g_{j} \quad \forall j \in N \cup P \\
& x_{a} \geq l_{a h r} \quad \forall a \in M_{A}, h \in M_{H}, r \in M_{R} \\
& y_{h} \geq l_{a h r} \\
& \forall a \in M_{A}, h \in M_{H}, r \in M_{R} \\
& z_{r} \geq l_{a h r} \\
& \forall a \in M_{A}, h \in M_{H}, r \in M_{R} \\
& x_{a}+y_{h}+z_{r}-l_{a h r} \leq 2 \quad \forall a \in M_{A}, h \in M_{H}, r \in M_{R} \\
& u_{j} \geq g_{j} \\
& \forall j \in N \cup P \\
& b_{j} \geq g_{j} \\
& \forall j \in N \cup P \\
& u_{j}+b_{j}-g_{j} \leq 1 \\
& \forall j \in N \cup P \\
& f_{j} \in\{0,1\} \\
& \forall j \in N \cup P \\
& x_{a} \in\{0,1\} \\
& \forall a \in M_{A} \\
& y_{h} \in\{0,1\} \\
& \forall h \in M_{H} \\
& z_{r} \in\{0,1\} \\
& \forall r \in M_{R} \\
& l_{a h r} \in\{0,1\} \\
& \forall a \in M_{A}, \forall h \in M_{H}, \forall r \in M_{R} \\
& u_{j} \in\{0,1\} \\
& \forall j \in N \cup P \\
& v_{j a} \in\{0,1\} \\
& \forall j \in N \cup P, \forall a \in M_{A} \\
& b_{j} \in\{0,1\} \\
& \forall j \in N \cup P \\
& g_{j} \in\{0,1\}
\end{aligned}
$$


In the objective function (9), we maximize the weighted combination of first and backup coverage given to crash nodes and paths; this is given in parenthesis. We add the $\varepsilon$ term to the objective function to ensure that, if there is at least one air ambulance located to cover a given node/path $j$, then $u_{j}$ should be 1 to relax the constraint (14), which locates at least two ground ambulances covering node/path $j$. Constraint (10) says that the budget allocated among the located EMS servers cannot exceed $B$. Constraint set (11) defines the first coverage variable. A motor vehicle crash location is covered if and only if it is covered at least once through ground, air, or joint ground-air. Constraint sets (12), (13) and (14) are backup coverage constraints. When there is at least one air ambulance that covers a given node/path $j$, then constraint (12) applies through the introduction of the $\varepsilon$ term in the objective function. When there is no air ambulance covering a given node/path $j$, then constraints (13) and (14) apply to ensure that at least two different ambulances will be located. The remaining constraints are the linearization constraints and binary variable definitions.

In practice, $\theta$ is determined by the service providers as a discretionary input between 0 and 1 . If both first and backup coverage are equally important, then $\theta$ should be set to 0.5 . On the other hand, if providing first coverage to as many crash locations as possible has a higher priority than providing backup coverage to some of the crash nodes and paths, then $\theta$ should be set close to 1 . Additionally, input parameter $d_{j}$ - weight attached to node/path $j$, is also discretionary. If service providers prefer to maximize number of nodes/paths covered by EMS servers, then $d_{j}$ should be 1 for all nodes and paths. However, in practice, there may be some locations where crashes occur more frequently than other crash nodes/paths. In this case, $d_{j}$ could be based on the number or density of crashes expected to occur at or near $j$.

\subsection{Solution Method}

For the solution of larger instances of MCGBM, we developed a greedy heuristic as we did for SCBM. In addition, we used CPLEX to get exact results on the smaller instances of MCGBM. The parameters of the greedy heuristic are as follows:

- $G$ : Greedy output, i.e. final locations of the air and ground ambulances and transfer points

- $\quad C$ : Total cost of located EMS servers

- $w(G)$ : Total weighted coverage obtained by the Greedy solution 
- $\quad C C_{j}=\left\{\begin{array}{cc}0, & \text { if } j \text { is fully covered } \\ 1-\theta, & \text { if } j \text { is covered just once and with a ground ambulance or combination } \\ 1, & \text { if } j \text { is uncovered }\end{array}\right.$

Greedy Heuristic Algorithm:

Step 0. $G=\varnothing, C=0, C C_{j}=1$ for all $j \in N \cup P, U=M_{A} \cup M_{H} \cup M_{A \cdot H \cdot R}$ and $w(\mathrm{G})=0$.

Step 1. For all $i \in U$, compute

$$
\begin{aligned}
& \text { ratio }(i)=\frac{w(i)}{c_{i}} \\
& w(i)=f((\text { Number of covered crash nodes/paths } j \text { when combination } i \text { is located }) \\
& \left.\left.\quad \text { multiplied by (Node/path weights } d_{\mathrm{j}}\right)\right)
\end{aligned}
$$

Evaluation of function $f$ changes with $i$ being a ground or air ambulance, or a combination of two.

If $i$ is a ground ambulance or a combination of a ground and an air ambulance, then only

$\theta * 100 \%$ of a given crash node/path is considered as covered due to backup need.

Let $k$ be the combination that maximizes ratio $(i)$ :

$$
k=\arg \max \operatorname{ratio}(i)
$$

Step 2. If $C+c_{k} \leq B$, then

$$
\begin{aligned}
& G \leftarrow G \cup k \\
& w(G) \leftarrow w(G)+w(k) \\
& C \leftarrow C+c_{k}
\end{aligned}
$$

Update location decision variables for $k$.

If $k$ is an air ambulance,

Delete covered crash nodes/paths when $k$ is located.

Set $C C_{j}=0$ for every $j$ covered by locating $k$.

If $k$ is a ground ambulance,

For uncovered crash nodes/paths $j$ that are covered by locating $k$,

Update $C C_{j}$ to $1-\theta$,

Record the ground ambulance index that provides first coverage to $j$, i.e.

to assign a different ambulance for backup coverage.

For crash nodes/paths $j$ with $C C_{j}=1-\theta$, which are covered by locating $k$,

Set $C C_{j}$ to 0 if $k$ is different than the ground ambulance that provides first coverage to $j$. 
Delete the covered crash nodes/paths.

If $k$ is a combination of a ground ambulance and an air ambulance,

Then apply same method with $k$ being a ground ambulance.

$U \leftarrow U-k$

Step 3. If $U \neq \varnothing$,

Return to Step 1.

Else, stop.

\subsection{Maximal Cover with a Given Number of Facilities of Each Type (MCGNFM)}

Instead of spending a limited budget to design a new EMS system, service providers may want to reorganize a current system with a given number of EMS servers of each type. The problem of finding a maximal cover with a given number of facilities of each type, i.e. ground ambulances, air ambulances and transfer points, can be modeled in a manner very similar to MCGBM. Therefore, we do not include a separate section on MCGNFM to give the formulation and solution method. In the formulation of MCGNFM, the budget constraint of MCGBM is replaced by three constraints that limit the number of ground ambulances, air ambulances and transfer points to be located. For its solution, a similar greedy heuristic can be used, in which instead of recording the budget that is spent on located EMS servers, we keep track of the number of servers that are located.

\section{Computational Studies}

To test the performance of the greedy heuristics, we generated both small and large problem instances for SCBM and MCGBM using an instance generator coded in $\mathrm{C}++$. Inputs of the instance generator are $x$ and $y$ coordinates of crash nodes and the two end points of the crash paths. The user may also assign weights to crash nodes and paths. Other inputs are the size of the study region, on-scene and off-scene times of ground and air ambulances, transferring time of the patient from a ground ambulance to an air ambulance, maximum out-of-hospital time and travel time, ground and air ambulance speeds, and TC locations. The outputs are the potential ground ambulance, air ambulance and transfer point locations, and node and path coverage matrices for ground and air ambulances, and joint coverage of the two. Potential EMS locations can be generated either randomly or following a spatial distribution similar to distribution of crash nodes and paths. 


\subsection{Generating coverage matrices}

Define:

$t_{i j}$ : travel time of EMS server $i$ from its home location to crash node/path $j$

$t_{j_{i} k}:$ travel time of EMS server $i$ from crash node/path $j$ to a location $k$

$c:$ location of the closest trauma center to the crash location

$B_{A_{1}}$ : on-scene time for a ground ambulance $B_{A_{2}}$ :off-scene time for a ground ambulance

$B_{H_{1}}$ :on-scene time for an air ambulance $\quad B_{H_{2}}$ : off-scene time for an air ambulance

$B_{t r}$ : transfer time of patient from a ground ambulance to an air ambulance

$T_{R}$ : pre-specified maximum travel time for a server(s) to arrive at trauma scene

$T_{H}$ : pre-specified maximum out-of-hospital time for a server(s) to get the patient to the closest TC

GROUND COVERED: If $t_{a j} \leq T_{R}$ and $t_{a j}+t_{j_{a} c}+B_{A_{1}}+B_{A_{2}} \leq T_{H}$ for a given $a$ and $j$

$$
\left(a \in M_{A}, j \in N \cup P\right) \text {, then } A_{a j}=1 \text {, otherwise it is } 0 \text {. }
$$

AIR COVERED: If $t_{h j} \leq T_{R}$ and $t_{h j}+t_{j_{h} c}+B_{H_{1}}+B_{H_{2}} \leq T_{H}$ for a given $h$ and $j$

$\left(h \in M_{H}, j \in N \cup P\right)$, and if $j$ is a permissible location for a helicopter to land at, then $A_{h j}=1$, otherwise it is 0 .

JOINT GROUND-AIR COVERED: If $\max \left(t_{a j}+t_{j_{a} r}, t_{h r}\right) \leq T_{R}$ and

$$
\begin{aligned}
& \max \left(t_{a j}+t_{j_{a} r}+B_{A_{1}}, t_{h r}\right)+t_{r_{h} c}+B_{t r}+B_{H_{2}} \leq T_{H} \text { for a given } a, h, r \\
& \text { and } j\left(a \in M_{A}, h \in M_{H}, r \in M_{R}, j \in N \cup P\right) \text {, then } A_{a h r j}=1,
\end{aligned}
$$

otherwise it is 0 .

The above coverage conditions, which are based on both travel time to crash scene and out-ofhospital time limits, are flexible. EMS providers may either focus on the travel time limit to crash scene, or on the total out-of-hospital time limit, or they may put conditions on both.

\subsection{Small Problem Instances}

To test the heuristic's performance, we generated 10 small sized problem instances. Instead of using the real costs of locating EMS servers, we used relative costs in terms of units. The location costs of the ground ambulances, air ambulances and transfer points are assumed to be 10 units, 50 units and 1 unit respectively. Study region is 10 by 10 miles. We generated 10 nodes and 
5 paths randomly, $1 \mathrm{TC}$ at the center of the study region, 30 potential ground ambulance locations following the spatial distribution of nodes and paths, 10 potential air ambulance locations randomly, and 36 transfer point locations on the corners of grids that have 2-mile intervals in each direction. Maximum out-of-hospital time for the patient is set to 45 minutes and maximum travel time to trauma scene is limited to 10 minutes. Ground ambulance and air ambulance speeds are in nautical miles/hr, and equal to $25 \mathrm{nmiles} / \mathrm{hr}$ and $120 \mathrm{nmiles} / \mathrm{hr}$, respectively.

From one problem instance to the other, we changed the locations of crash nodes and paths, and the potential locations of ground ambulances and air ambulances.

Figures 1 and 2 illustrate the results of two of the runs for the SCBM. Due to small study region and limited budget, only ground ambulances are located. Table 1 gives the average optimality gap and solution times. Since the numbers of crash nodes and crash paths are small, the optimality gap between the objective values of the Greedy heuristic and CPLEX is significantly large. Even for very small problem instances, finding the optimum solution using CPLEX requires a long run time.

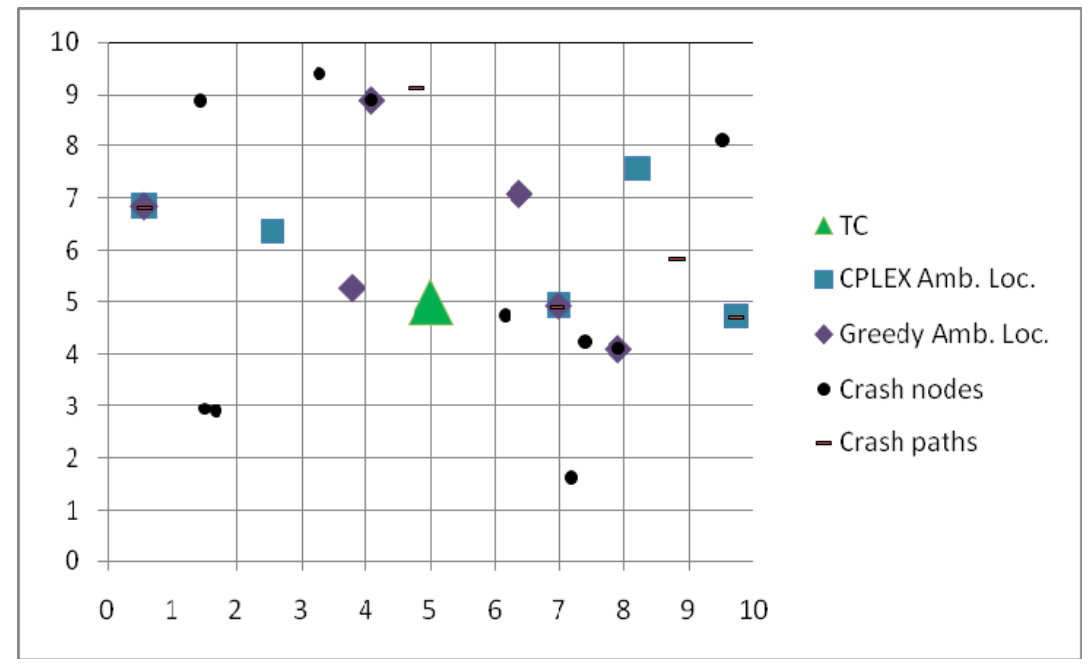

Figure 1. SCBM: Small example (Run 9) - \%16.7 optimality gap 


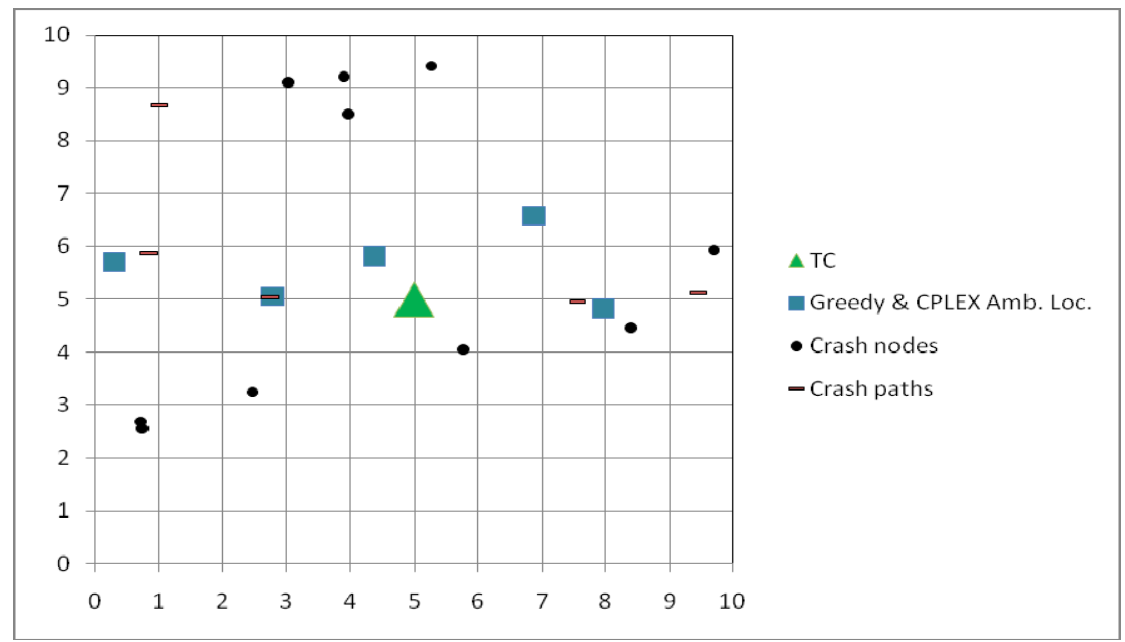

Figure 2. SCBM: Small example (Run 7) - \%0 optimality gap

Table 1. SCBM: Average optimality gap and solution times

\begin{tabular}{|r|r|r|}
\hline \multicolumn{2}{|c|}{ Greedy Heuristic } & \multicolumn{1}{c|}{ CPLEX } \\
\hline Average Optimality Gap & Average Solution Time & Average Solution Time \\
\hline $13.2 \%$ & $10.8 \mathrm{sec}$ & $23 \mathrm{mins}$ \\
\hline
\end{tabular}

For the MCGBM, we assigned the same weight for the nodes and paths. Table 2 shows the average run times and optimality gap for the greedy heuristic, CPLEX and premature CPLEX respectively, when solution time of CPLEX and premature CPLEX is fixed to 500 seconds. For the same problem instances as used in SCBM, three different theta $(\theta)$ values are used to analyze the sensitivity of the solution when we switch the priority between first $(\theta=1)$ and backup coverage $(\theta=0)$. Instead of using zero for the value of $\theta$, we used 0.01 , because if we give all the weight to backup coverage, the greedy heuristic never locates ground ambulances. When $\theta=0$, the backup coverage does not increase by locating a single ground ambulance. Since the greedy heuristic does not locate ground ambulances in pairs, locating an air ambulance is preferred at each iteration of the heuristic. In 14 out of 30 runs, CPLEX fails to find the optimum solution.

We also analyzed the best CPLEX parameter settings (premature CPLEX) for our problem. We tried several variable and node selection strategies in the branch and bound tree and allowed for backtracking. We selected different levels of probing and switched mixed integer program (MIP) emphasis from optimality to feasibility to find a feasible solution quickly. For $90 \%$ of the runs, premature CPLEX finds the optimal solution in less than 500 seconds. The average results show that the greedy heuristic has the smallest solution time and the quality of solutions are 
comparable with CPLEX results. However, with solution time fixed to 500 seconds, premature CPLEX provides the best solutions.

Table 2. MCGBM: Comparison of Greedy, CPLEX and Prem. CPLEX performances

\begin{tabular}{|r|r|r|r|r|r|}
\hline \multicolumn{2}{|c|}{ Average Solution Time (sec) } & \multicolumn{3}{c|}{ Average Optimality Gap (\%) } \\
\hline Greedy & CPLEX & Prem. CPLEX & Greedy & CPLEX & \multicolumn{1}{c|}{ Prem. CPLEX } \\
\hline 7.1 & 275.8 & 86.0 & 6.0 & 8.0 & 0.8 \\
\hline
\end{tabular}

\subsection{Large Problem Instances}

For the large problem instances, we generated 50 nodes and 20 paths randomly on a study region of size 40 by 40 miles. Two TCs are located at the centers of 20 by 40 mile grids, 80 potential ground ambulance locations are generated following the spatial distribution of nodes and paths, 16 potential air ambulance locations are generated at the centers of 10 by 10 mile grids, and 64 transfer point locations are generated at the centers of 5 by 5 mile grids. We used same time and speed parameter settings as with the small problem instances. Relative server location costs are also the same as with small sized problem instances.

We worked on five problem instances. As in the small problem instances, we changed node and path locations and potential ground ambulance locations from one problem instance to the other. For all the instances, SCBM turned out to be infeasible, since potential EMS server locations are limited and not enough to cover all crash nodes and paths while also ensuring backup coverage. Even though the problems are infeasible, greedy results on SCBM give us the approximate budget to be spent among the EMS servers in order to achieve a total coverage close to a set cover with backup.

For the MCGBM, we assumed the same weight for the nodes and paths. Three different theta values are used; 1 (all the importance given to first coverage), 0.5 (equal weights for first and backup coverage), and 0.01 (almost all weight is given to backup coverage). We compared the solutions of greedy heuristic, CPLEX and premature CPLEX with best MIP parameter settings for our problem. We used the same CPLEX parameter settings as in the small problem sizes, but decreased the level of probing since it takes too much time. In 14 out of 15 runs (93\%) CPLEX, and in 11 out of 15 runs (73\%) premature CPLEX failed to find an integer solution when their solution time is fixed to greedy solution time, which is on the average 398 seconds. For the few runs that CPLEX and premature CPLEX found a feasible solution, the objective value is worse than for the greedy solution. Since location decisions are strategic decisions, solution time of CPLEX and premature CPLEX are relaxed to 5 hours for the same large examples. In this 
case, both CPLEX and premature CPLEX find a feasible solution for all the runs. However, the average optimality gap for CPLEX and premature CPLEX are 8.6\% and 10.3\% respectively, and Greedy still provides a better solution for $20 \%$ and $40 \%$ of the runs as compared to CPLEX and premature CPLEX respectively. For the runs that the Greedy solution is worse, the improvement over the Greedy solution is on the average $2.7 \%$ by CPLEX and $2.8 \%$ by premature CPLEX. Considering that real life problem sizes are larger than the examples in this section, we prefer to use the developed greedy heuristic as the solution method for the large sized instances of MCGBM.

\section{Case Study}

To illustrate the applications of SCBM and MCGBM in designing ground and air EMS systems, we worked on a case study that uses motor vehicle crash data and emergency hospital data from the state of NM. Even if most of the parameter settings in the models are based on real data, some of the parameter requirements are based on the realistic estimations used previously in the computational studies (Section 6). Therefore, the case study results do not provide any guidance for a real reorganization of emergency resources for the state of NM.

In the previous paper by Tokar-Erdemir et al. (2008), a model that locates aeromedical servers in order to maximize coverage is applied to the state of NM and results are used to evaluate the crash node and path coverage performance of the existing eight aeromedical base locations in NM. This case study addresses a more complicated real-life problem and differs from the analysis in Tokar-Erdemir et al. (2008) as shown in Table 3.

Table 3. Case study analysis vs. analysis in Tokar-Erdemir et al. (2008)

\begin{tabular}{|l|l|}
\hline Tokar-Erdemir et al. (2008) & Case Study \\
\hline Maximize first coverage & $\begin{array}{l}\text { Maximize combination of first and } \\
\text { backup coverage }\end{array}$ \\
\hline Locate only air ambulances & $\begin{array}{l}\text { Locate ground ambulances, air } \\
\text { ambulances and transfer points } \\
\text { considering three coverage options }\end{array}$ \\
\hline Coverage is based on only response time & $\begin{array}{l}\text { Coverage is based on both response time } \\
\text { and total service time until the patient is } \\
\text { transferred to the closest TC }\end{array}$ \\
\hline $\begin{array}{l}\text { Maximize weighted coverage: Crash nodes } \\
\text { and paths are weighted such that more } \\
\text { importance is given to urban regions where } \\
\text { crashes occur more frequently }\end{array}$ & $\begin{array}{l}\text { Maximize number of covered crash nodes } \\
\text { and paths (unweighted) }\end{array}$ \\
\hline $\begin{array}{l}\text { Air ambulances are assumed to land } \\
\text { everywhere }\end{array}$ & $\begin{array}{l}\text { Air ambulances cannot land on crash } \\
\text { locations within driving distance of TCs } \\
\text { and 24-hour emergency hospitals }\end{array}$ \\
\hline
\end{tabular}


To better reflect a real life scenario, we also modified the coverage conditions in the models specific to the case in which ground ambulances are only allowed to go to crash scenes within their own jurisdictions. Therefore, ground coverage of crash nodes and crash paths is restricted to the ground ambulances within the same jurisdiction. However, we also analyzed the solution by relaxing the "same jurisdiction coverage" assumption. This latter case is critical for a disaster type scenario when all emergency resources could be shared by different jurisdictions. We compared the solutions of two situations to see the change in the type of selected EMS servers.

\subsection{Data}

Table 4 shows the parameter settings for the case study. The NM traffic accident data is derived from police accident reports and was provided to us by the Division of Government Research at the University of New Mexico. The traffic accident data is based upon an accident record file maintained by the state of New Mexico Highway and Transportation Department. The data file provides information on the location, severity, causes and the number of people killed/injured in motor vehicle crashes in the state of NM from year 2000 to 2003. We used the KABCO scale (Farmer, 2003) to identify the fatal (class K (killed)) and most severe injury crashes (class A (incapacitating injury)). This subset of crashes gave us a total of 1962 crash nodes.

Paths are based upon US Census Bureau TIGER 2000 street files. In our analysis, we chose crash paths as the interstate highway segments with 0.3 miles of length, which gives us a total of $6681 \mathrm{crash}$ paths to be covered.

Table 4. Parameter settings for the case study

\begin{tabular}{|c|c|}
\hline Number of Crash Nodes & 1962 \\
\hline Number of Crash Paths & 6681 \\
\hline Number of TCs & 6 \\
\hline Potential Ground Ambulance Locations & 178 \\
\hline Potential Air Ambulance Locations & 40 \\
\hline Potential Transfer Point Locations & 73 \\
\hline Maximum Out-of-Hospital Time & $45 \mathrm{mins}$ \\
\hline Maximum Travel Time to Scene & $10 \mathrm{mins}$ \\
\hline Ground Ambulance Speed & $25 \mathrm{nmiles} / \mathrm{hour}$ \\
\hline Air Ambulance Speed & $120 \mathrm{nmiles} / \mathrm{hour}$ \\
\hline
\end{tabular}


Since we are considering motor vehicle crashes, we assumed that the trauma victims are transported to the closest TC after initial emergency response at the crash scene. In our analysis, we considered one Level 1, one Level 2 and four Level 3 TCs in NM. These TCs are located in five different counties. In selecting potential locations for ground ambulances, we included as candidate sites the locations of hospitals with 24-hour emergency rooms. The list of NM hospitals is developed from multiple sources including American Hospital Directory website and New Mexico Department of Health website, and telephone contacts of individual hospitals to verify that they had 24-hour emergency rooms.

The 178 potential ground ambulance locations are distributed within the 33 counties of NM based on their relative population. Thus for higher population areas, we have more potential ground ambulance locations. In 21 counties, there is a hospital with a 24-hour emergency room, so we generated at least two potential ground ambulance locations at these hospitals and the remaining potential locations are randomly placed among the crash nodes.

The 40 potential air ambulance locations are the combinations of the 8 current aeromedical bases in NM, plus 16 optimal aeromedical base locations suggested by results of our previous work (Tokar-Erdemir et al., 2008), plus an additional 16 potential aeromedical base locations on evenly distributed grid points with 100-mile grid intervals in each direction.

For the 73 potential transfer point locations, we used the same locations with air ambulances (40 transfer points), and we also used grid points with 50-mile grid intervals in each direction (33 transfer points).

For the air ambulance landing zones, we assumed that helicopters would not land at crash locations within driving distance of a 24-hour emergency hospital (28 hospitals). The driving distance is assumed to be a 5-mile radius. Our analysis showed that $48 \%$ of the crash nodes and $10 \%$ of the crash paths are within driving distance to these hospitals.

Since the case study is presented here to illustrate the capabilities of the developed models, we did not use real cost values for the EMS servers. Instead, we used relative costs of 10 units, 50 units and 1 unit for ground ambulances, air ambulances and transfer points, respectively.

\subsection{Results}

The SCBM is not feasible for the case study with the selected parameter settings. The number of potential EMS server locations is insufficient to provide coverage to all crash nodes and paths and also ensure backup for required regions.

For the MCGBM, we selected two budget levels, 100 and 400 units. We did not go over 400 units due to computational limitations. We used three different theta $(\theta)$ values: 1 (all weight 
given to first coverage), 0.5 (same importance for first and backup coverage) and 0.01 (almost all weight given to backup coverage).

For 100 units of budget level, Tables 5 and 6 respectively show the MCGBM results with and without the jurisdiction limitation. The same information is given in Tables 7 and 8 for 400 units of budget level, but for theta $(\theta)$ values of 1 and 0.5 . As the tables show, with an increasing budget level, the total coverage provided to crash nodes and crash paths also increases when we keep other parameters constant. When the importance given to backup coverage increases, (i.e. decreasing theta), first coverage provided to crash nodes and paths decreases as expected. However, since the number of resources that can be located is small for 100 units of budget level, due to using a greedy heuristic approach, the backup coverage percentage decreases even though the backup weight increases. The greedy heuristic does not locate ground ambulances when theta $=0.01$, since the importance is on backup coverage. However, instead of locating initially air ambulances, if we located ground ambulances in pairs, then the total coverage that we had achieved could be higher. This is the disadvantage of the greedy heuristic. However, in real life, we do not expect the service providers to give all the importance to backup coverage, therefore giving the priority to backup coverage $(\theta<0.5)$ does not seem reasonable. In Tables 5 and 6 , adding a jurisdiction limitation for travel boundaries of ground ambulances decreases coverage levels as compared to the coverage levels in Tables 7 and 8, which is intuitive.

Table 12. MCGBM case study results: Budget $=100$ units and with jurisdiction limits

\begin{tabular}{|c|c|c|c|c|c|}
\hline \multirow[b]{3}{*}{ Theta $(\theta)$} & \multicolumn{5}{|c|}{$\begin{array}{c}\text { Budget }=100 \text { units } \\
\text { With County Limit for Ambulances }\end{array}$} \\
\hline & \multicolumn{3}{|c|}{ Number of Located EMS } & \multicolumn{2}{|c|}{\begin{tabular}{|c|} 
Percentage of \\
Nodes and Paths with \\
\end{tabular}} \\
\hline & Ambulances & Helicopters & $\begin{array}{c}\text { Transfer } \\
\text { Points }\end{array}$ & $\begin{array}{c}\text { First } \\
\text { Coverage }\end{array}$ & $\begin{array}{l}\text { Backup } \\
\text { Coverage }\end{array}$ \\
\hline 1 & 5 & - & ( & $9.7 \%$ & N/A \\
\hline 0.5 & 5 & 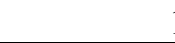 & ( & $8.3 \%$ & $6.8 \%$ \\
\hline 0.01 & 0 & 2 & ( & $2.7 \%$ & $2.7 \%$ \\
\hline
\end{tabular}

Table 13. MCGBM case study results: Budget $=100$ units and no jurisdiction limits

\begin{tabular}{|r|r|r|r|r|}
\cline { 2 - 6 } \multicolumn{1}{c|}{} & \multicolumn{4}{c|}{ Budget = 100 units } \\
No Jurisdiction Limit \\
\hline
\end{tabular}


Table 14. MCGBM case study results: Budget $=400$ units and with jurisdiction limits

\begin{tabular}{|r|r|r|r|r|r|}
\cline { 2 - 6 } \multicolumn{1}{c|}{} & \multicolumn{4}{c|}{ Budget $=400$ units } \\
\hline \multirow{3}{*}{ Theta $(\theta)$} & \multicolumn{3}{|c|}{ Number of Located EMS } & \multicolumn{2}{c|}{$\begin{array}{c}\text { Percentage of } \\
\text { Nodes and Paths with }\end{array}$} \\
\cline { 2 - 6 } & Ambulances & Helicopters & $\begin{array}{c}\text { Transfer } \\
\text { Points }\end{array}$ & $\begin{array}{c}\text { First } \\
\text { Coverage }\end{array}$ & $\begin{array}{c}\text { Backup } \\
\text { Coverage }\end{array}$ \\
\hline 1 & 5 & 7 & 0 & $13.9 \%$ & N/A \\
\hline 0.5 & 5 & 7 & 0 & $12.5 \%$ & $11.0 \%$ \\
\hline
\end{tabular}

Table 15. MCGBM case study results: Budget $=400$ units and no jurisdiction limits

\begin{tabular}{|r|r|r|r|r|r|}
\cline { 2 - 6 } \multicolumn{1}{c|}{} & \multicolumn{5}{c|}{ Budget $=400$ units } \\
No Jurisdiction Limit \\
\hline \multirow{3}{*}{ Theta $(\theta)$}
\end{tabular}

\section{Conclusions}

In this paper, we presented three coverage types; sending only a ground ambulance, sending only an air ambulance, or providing joint coverage using a combination of a ground and an air ambulance in which the two rendezvous and the patient is transferred from the ground ambulance to the air ambulance at a transfer point. A crash node or path is said to be covered if it is within a pre-determined response time limit from one of the located EMS servers and also within a pre-determined service time limit, so that the trauma victim could be transported to the closest TC by any one of the coverage methods within the specified time limit. Joint coverage through a transfer point and coverage definition based on two criteria are new to the locationcoverage literature.

Considering the three coverage options, two sets of models (SCBM and MCGBM) as variants of LSCP and MCLP are introduced to recommend optimal ground ambulance, air ambulance and transfer point locations. These models also address uncertainty in spatial distribution of motor vehicle crash locations and consider unavailability of ground ambulances by ensuring backup coverage when needed.

A greedy heuristic is proposed for the solution of both models. The greedy heuristic provides high quality solutions much faster than CPLEX. Development of better heuristic methods is left as a future work. 
The results are illustrated on a case study that uses crash and emergency hospital data from the State of NM. Results are provided for different combinations of first and backup coverage weights under two different budget levels. A comparison is presented for the cases where ground ambulances could only be dispatched to crash locations within their own jurisdiction and when the ground ambulances could be utilized anywhere in the entire study region. Results show that optimal coverage percentages decrease when ground ambulance are restricted to be dispatched only within their own jurisdiction.

The key implication of this paper is that co-operative coverage models can be beneficial for situations where using both server types improves service levels. The key policy implication of this paper is that traditional ambulance services can provide a valuable supplement to air ambulance services when responding to trauma-related injuries; this is done via direct coverage by traditional ambulance services of calls that originate close to a trauma center and by cooperative coverage of calls that occur where an air ambulance cannot land directly. The concepts of the paper could be applied to other contexts where joint coverage is relevant, e.g. coverage of ground military units by secondary units that provide supplies when they run out.

\section{Discussion}

We close the paper by providing a discussion on (a) coverage types, (b) unavailability of EMS servers, and (c) area coverage.

\subsection{Coverage types}

In the paper, the joint coverage option is considered to be used only for the crash locations that air ambulances cannot land at, because for the crash nodes and paths that air ambulances can land, the out-of-hospital time with joint coverage is expected to be longer. However, if the ground ambulance and the air ambulance meet at the crash scene - i.e. no scene landing issue, then joint coverage may be more advantageous both in terms of shorter out-ofhospital time and/or providing higher medical care at the crash scene. Exploring this fourth type of coverage in the models is an opportunity for future work.

The type of EMS response actually provided to a crash location may also depend on the severity of the crash. For most crashes, ground ambulances typically provide the first response. If the injuries are serious and the accident victim requires rapid transportation to a $\mathrm{TC}$, then air ambulances are subsequently requested by responders at the scene. Therefore, the optimal locations of ground ambulances, air ambulances and transfer points can depend on the severitybased spatial distribution of accidents. This is another opportunity for further work. 


\subsection{Unavailability of EMS servers}

The two models developed in this paper addresses unavailability of ground ambulances through the assignment of backup EMS servers. In this way, when one of the ground ambulances is busy when requested, then the backup may respond to the crash scene. Since the problem of joint ground-air coverage is complex to model, we used a backup coverage approach to handle unavailability of ground ambulances. However, this approach does not always guarantee timely response to a crash location. While in high crash density regions, several backups may be needed, in low crash density regions, assigning a backup may be unnecessary. Precise modeling of backup options in this context is another opportunity for future work.

\subsection{Area coverage}

If the spatial distribution of accidents that require emergency response can be only on a given set of paths - such as motor-vehicle crashes that occur on roads, then the location-coverage models developed here are appropriate. However, both ground and air EMS providers usually respond to a wide range of accidents, which are spatially distributed over a region. Therefore, if we focus on all types of emergency situations, then we should consider area coverage instead of path coverage. The accidents may occur anywhere in a given region, and each part of the study region may have a different weight indicating likelihood of accident occurrence. Area coverage consideration requires further investigation and is suggested as a future research opportunity.

\section{References}

American Hospital Directory website, $<$ http://www.ahd.com/freelist.php3?mname $=\&$ mcity $=\& m s t a t e ~ \% 5 B \% 5 \mathrm{D}=\mathrm{NM} \& \mathrm{mzip}=\& \mathrm{mphon}$ $\mathrm{e}=$ \&submitted $=$ Search $>$.

Atkinson, J.B., Kovalenko, I.N., Kuznetsov, N., Mykhalevych, K.V., 2006. Heuristic methods for the analysis of a queuing system describing emergency medical service deployed along a highway, Cybernetics and Systems Analysis 42 (3), 379-391.

Atkinson, J.B., Kovalenko, I.N., Kuznetsov, N., Mykhalevych, K.V., 2008. A hypercube queuing loss model with customer-dependent service rates. European Journal of Operational Research 191, 223-239.

Bianchi, G., Church, R.L., 1988. A hybrid fleet model for emergency medical design. Social Science and Medicine 26, 163-171.

Branas, C.C., MacKenzie, E.J., ReVelle, C.S., 2000. A trauma resource allocation model for ambulances and hospitals, Health Services Research 35 (2), 489-507.

Branas, C.C., ReVelle, C.S., 2001. An iterative switching heuristic to locate hospitals and helicopters, Socio-Economic Planning Sciences 35, 11-30.

Brotcorne, L., Laporte, G., Semet, F., 2003. Ambulance location and relocation models. European Journal of Operational Research 147, 451-463. 
Chelst, K., Barlach, Z., 1981. Multiple unit dispatches in emergency services" models to estimate system performance, Management Science 27 (12), 1390-1409.

Church, R.L., Gerrard, R.A., 2003. The multi-level location set covering model. Geographical Analysis 35, 277-289.

Church, R.L., ReVelle, C., 1974. The maximal covering location problem. Papers of the Regional Science Association 32, 101-118.

Daskin, M.S., 1995. Network and Discrete Location: Model Algorithms and Applications. John Wiley and Sons Inc, New York.

Daskin, M.S., Stern, E.H., 1981. A hierarchical objective set covering model for emergency medical service vehicle deployment. Transportation Science 15, 137-152.

Eaton, D.J., Daskin, M.S., Simmons, D., Bulloch, B., Jansma, G., 1985. Determining emergency medical deployment in Austin, Texas. Interfaces 15 (1), 96-108.

Farmer, C.M., 2003. Reliability of police-reported informationfor determining crash and injury severity. Traffic Injury Prevention 4 (1), 38-44.

Hogan, K., ReVelle, C.S., 1986. Concepts and applications of backup coverage. Management Science 34, 1434-1444.

Iannoni, A.P., Morabito, R., 2007. A multiple dispatch and partial backup hypercube queuing model to analyze emergency medical systems on highways. Transportation Research E 43, $755-771$.

Karp, R.M., 1972. Reducibility among combinatorial problems. In: Complexity of Computer Computations, R.E. Miller and J.W. Thatcher (eds.), Plenum Press, New York, 85-104.

Larson, R.C., 1974. A hypercube queuing model for facility location and redistricting in urban emergency services, Computers and Operations Research 1, 67-95.

Lerner, E.B., Bilittier, A.J., Sikora, J., Moscati, R.M., 1999. Use of a geographic information system to determine appropriate means of trauma patient transport. Academic Emergency Medicine 6 (11), 1127-1133.

Marianov, V., ReVelle, C.S., 1992. A probabilistic fire protection siting model with joint vehicle reliability constraints. Papers of the Regional Science Association 71 (3), 217-241.

Marianov, V., ReVelle, C.S., 1995. Siting emergency services. In: Drezner, Z. (Ed.), Facility Location. Springer, New York, pp. 199-223.

Mendonça, F.C., Morabito, R., 2001. Analyzing emergency service ambulance deployment on a Brazilian highway using the hypercube model, Journal of the Operational Society 52, 261-270.

New Mexico Department of Health website, <http://www.health.state.nm.us/>.

Schilling, D., Elzinga, D.J., Cohon, J., Church, R., ReVelle, C., 1979. The TEAM / FLEET models for simultaneous facility and equipment siting, Transportation Science 13, 163-175.

Tokar Erdemir, E., Batta, R., Spielman, S., Rogerson, R., Blatt, A., Flanigan, M., 2008. Location coverage models with demand originating from nodes and paths: Application to cellular network design. European Journal of Operational Research 190, 610-632.

Tokar Erdemir, E., Batta, R., Spielman, S., Rogerson, R., Blatt, A., Flanigan, M., 2008. Optimization of aeromedical base locations in New Mexico using a model that considers crash nodes and paths. Accident Analysis and Prevention 40, 1105-1114.

Toregas, C.R., Swain, R., ReVelle, C.S., Bergman, L., 1971. The location of emergency service facilities. Operations Research 19, 1363-1373. 